\title{
Conceptualizing Fidelity for HCI in Applied Gaming
}

\author{
Heide Lukosch ${ }^{1}$, Stephan Lukosch ${ }^{1}$, \\ Simon Hoermann ${ }^{2}$ and Robert W. Lindeman ${ }^{3}$ \\ ${ }^{1}$ Delft University of Technology, Jaffalaan 5, 2628 BA Delft, Netherlands \\ ${ }^{2}$ University of Canterbury, Engineering Road, Ilam,8041 Christchurch, New Zealand \\ ${ }^{3}$ HIT Lab NZ, 2 Engineering Road, Ilam, Christchurch, New Zealand \\ \{h.k.lukosch $\{$ s.g.lukosch $\}$ atudelft.nl; \\ simon.hoermann@canterbury.ac.nz; gogo@hitlabnz.org
}

\begin{abstract}
Fidelity of games as a concept describes the level of representation of, or accordance with reality. The level of fidelity has influence on the interaction between player and game. Our study discusses the outcomes of a literature study and three cases with the goal to propose a comprehensive framework of game fidelity. This framework could help game designers and researchers to adopt the 'right' or sufficient level of fidelity to achieve the intended objectives related to applied games. Our results show that functional and psychological fidelity have a higher impact on the experience and effects of applied games than their physical fidelity. Social and ethical fidelity are proposed as new dimensions of game fidelity that still have to be explored. In literature, both low and high levels of fidelity are described as effective in applied games, while a medium level seems not to be beneficial for the player.
\end{abstract}

Keywords: Applied Games, Fidelity, Human Computer Interaction

\section{$1 \quad$ Introduction}

In this paper, we discuss necessary factors leading to the development of a new conceptual framework of game fidelity for digital, applied games. One of the vital theoretical issues in applied games research is the question about how to make connections between the game world and the 'real world'. This connection is bidirectional: from the game to the real world in the form of affective action, and from the real world to the game in the form of realistic representation (Galloway, 2004). Failure to achieve the 'right' level of realism in a training environment such as an applied game, might result in false learning when applied to reality (Chalmers, Debattista \& Ramic-Brkic, 2009).

In comparison to images, where the question related to the representation of reality addresses notions of mimetic mirroring of reality, games additionally inherit the concept of action (Galloway, 2004). As games require constant input from the player, a realistic game must be realistic in doing, in action (Aarseth, 1997), and not only in its physical representation of the reality. Our proposed concept of game fidelity follows the paradigm in HCI that focuses on the quality of interaction, and the nature of meaning and meaning creation (Harrison, Tatar \& Sengers, 2007) in the interaction between player and game. As such, game fidelity contributes to the engagement of a player with a game whereas engagement can be seen as desire and willingness to stick to a game for longer, and represents the lowest-level of involvement with a game towards immersion (Kayali et al., 2018; Brown \& Cairns, 2004). The feeling of being in places in games is furthermore related to the concept of presence, which can be 
subdivided into experiences of psychological immersion (or psychological fidelity), perceived naturalness (or physical fidelity), and spatial awareness, or engagement (Lombard \& Ditton, 1997).

Based on related research, we propose to approach the action-related representation of reality in digital, applied games along the following four dimensions:

1. Physical fidelity is the dimension that is most widely described in research so far (Harteveld, 2011), the most notable example being visual fidelity. It is defined as the degree to which the virtual simulation emulates the physical properties of the target environment (Alexander et al., 2005).

2. Functional fidelity or action-related representation of reality, describes the need for realistic actions and functions that the game allows. It is the extent to which the virtual tools and devices react as the real tools to the tasks carried out by the users in the simulation (Alexander et al., 2005).

3. Psychological fidelity relates to activity, but not so much the physical activity as the cognitive and psychological dimensions of it, such as realistic levels of emotions, engagement, and stress. It is the level at which the virtual simulation is able to elicit affective states similar to real world experiences (Alexander et al., 2005).

4. Social fidelity in an age of $\operatorname{MOO}(\mathrm{RP})$ games points out that the realistic representation of social interaction and connection in games is still an underrepresented topic in games research. As games are an active medium, they require the social reality of the player and the social reality of the game (Galloway, 2004). It describes how well the simulation is able to mimic real world social interactions.

We conceptualize the forms of realistic representation, or game fidelity with regard to designing games that foster human computer interaction and their contribution to creating meaning of and engagement with applied games. In this sense, we define game fidelity as a continuum, ranging from low levels of fidelity to high levels of fidelity. Our research approach is twofold. First, we report on the outcomes of a literature study to discuss existing approaches towards game fidelity and related concepts such as engagement, immersion, and presence. Secondly, we discuss various possibilities that can influence the fidelity of applied games and their effect on presence and target outcomes. With a cross-case analysis based on some of our recent results, we explore how to balance the four aspects of fidelity in applied games to produce effective outcomes in terms of presence and engagement in applied games. We conclude with a first step towards a comprehensive model of game fidelity.

\section{Fidelity in Applied Games: Current Research and Gaps}

In high fidelity design for both entertainment and applied games, technologies such as augmented reality (AR) can address all human senses, i.e., sight, sound, smell, taste and touch. Users can become aware of each other's activities by using AR environments for spatial remote collaboration (Lukosch et al., 2015). Yet, one challenge seems to be to create a realistic interaction between real objects and virtual objects (Schraffenberger \& Van der Heide, 2013). Another important challenge lies in the right balance between fidelity, design effort, and expected outcome of an applied game. To evaluate the right balance, we are working towards a better understanding of the aspects of game fidelity, 
and propose a framework of these elements that altogether creates the fidelity, or realism in applied games.

With the advance of AR and VR technologies, the possibilities to improve the realism in applied games has increased. The type of relationship between reality and games we are referring to in our work is called interaction fidelity. Interaction fidelity refers to the degree of exactness with which real-world interactions are reproduced in the game (McMahan, 2011). Virtual training, one of the purposes of applied gaming, is seen as most effective when interactions show a high level of fidelity (Waller, Hunt, \& Knapp, 1998). For presence, engagement, and usability, a study found that both display and interaction fidelity had significant positive effects (McMahan, Bowman, Zielinski, $\&$ Brady, 2012). Further studies show that transfer from what has been learned within a simulation or game to the real world is one of the main reasons to use high fidelity (VR) environments (Chalmers, Debattista \& Ramic-Brkic, 2009; Bhargava et al., 2018).

In a study carried out by Bhargava et al. (2018), based on various tests, the authors show that high and low fidelity interactions can lead to better results of task performance, compared to mid-level fidelity. Yet, the different levels of fidelity had no significant impact on the learning outcomes. The tests included in their study were mainly focused on task and visual fidelity, which we would call physical and functional fidelity. Social and psychological fidelity has not been addressed in this work. Other research has shown that failure to achieve the 'right' level of realism holds the risk that the player adopts a 'wrong' or different strategy than needed in real life (Chalmers \& Debattista, 2009).

A multi-level study carried out by Chalmers \& Debattista (2009), discuss the importance of multi-sensory stimuli in virtual environments when used for training purposes. One important finding they report on is that the more familiar an environment is to the player, the less he or she is paying attention. In addition, it is noted that there might be multiple interrelations between the senses addressed by any virtual environment. Recommendations from this study include that in designing a highfidelity virtual environment, the parts of the environment that are vital for the experience should show a high level of fidelity, while others might show lower levels of fidelity without significant impact on the effectiveness of the virtual environment. Yet, their findings again mainly address the functional and physical fidelity, but do not include considerations with respect to psychological or social fidelity.

Cornacchione (2012) discusses the relationship between fidelity and training outcomes across different media and time. The results of his study with different levels of fidelity in a simulation environment used for learning imply that students tend to welcome learning strategies that show a higher level of functional and physical fidelity. Such higher levels of fidelity were also related to better training transfer from simulation to reality. Contrary to these results, Feinstein \& Cannon (2002) show that many studies exploring the relationship between fidelity and learning found that lower fidelity actually can assist in acquiring the details of training and education (Alessi, 1988; Dwyer, 1974; Gagne, 1954; Miller, 1974). Hays \& Singer (1989) state that it might be effective to move away from reality in order to increase learning effects.

On a more conceptual level, Harteveld (2011) defines three criteria for realism in game play: validity, flexibility and fidelity. In terms of validity, which he defines as the visual resemblance between reality and virtual worlds for training, much progress has been made in the past decades. With the increasing possibilities of high-speed graphical visualization, applied games have become more and more realistic. Applied games with 
adequate levels of detail for buildings and objects are readily available. Flexibility can be guaranteed by modular design of the games, the use of scenario editors, and by teacher-led games. Through applied games, different virtual scenarios can be designed and played.

An overview of literature on the relationships between fidelity and effect of virtual, game-based learning environments shows that results are often contradictory. These differences in findings can partly be explained by the distinctive understanding of the concept of fidelity the authors apply. This is the main reason why we propose a multilayered concept of fidelity for applied gaming. Below, we first discuss findings from our own work, then propose a framework of applied game fidelity based on both our literature review and case study results.

\section{Cross-Case Analysis: Applications in Safety, Security and Health}

In this section, we illustrate the design process and effects of applied games through three different cases. The first case relates to a study in the safety and security domain using a game in a VR environment with the goal of training team situation awareness and communication. The second case reports on experiences with an AR game enforcing information asymmetry in the security domain to study collaboration and communication behavior of geographically distributed team members.

\subsection{CharliePapa: VR Game for Police Training}

In this case study, a high-fidelity virtual reality (VR) environment was developed and tested for its usefulness in police team training. Expected outcomes of this training are defined as improved situation awareness and communication skills in reconnaissance teams. For this study, three different aspects of fidelity were identified. First, the applied game represents assignments, which are described in terms of functional fidelity. Functional fidelity refers to the degree in which the roles, processes and tasks of the agents and the players match with roles, processes and tasks of the reference system of the game, mostly the "reality". In the VR simulation game, a police officer from the Netherlands is able to take over the role of a police officer of any rank the Dutch police offer and fit it to the tasks assigned in the game. The player is able to move around, represented by a virtual avatar in the game world, and is able to look, hear, communicate, and take appropriate action. The task in the game is based on a task description by a Dutch police officer of the rank and function that is represented in the game, for example the daily route of a surveillance team. This refers to the notion of functional fidelity.

Secondly, the surrounding in which the game takes place, its objects and avatars, are related to physical fidelity. This level of fidelity refers to the degree in which the environment of the game, the objects within the game world including their textures, color and movements, the agents in the game and their behaviors, and the sound match with the reference system of the game, mostly the "reality". For example, the computeraided representation of a tree in this VR game has a high degree of audio-visual fidelity as its texture including shadow and light have a very natural look, corresponds properly with its environment regarding color and size, and its blossoms move with the circulation of the air. 
Thirdly, emotions, such as stress, joy, anger, or doubt, that a player feels by accomplishing his or her tasks are termed psychological fidelity. Psychological fidelity refers to the degree in which the emotional and cognitive reactions of the player match with those in reality. It includes the perception of the game play process, the feeling of flow and experience of immersion within the game. For example, police officers experience stress when they face a dangerous situation, where important decisions have to be taken within seconds. The VR game includes high psychological fidelity as players have reported that they experience "realistic" stress and time pressure while carrying out their assignments in the game.

Following the three aspects of fidelity focused on in this study, we addressed them throughout the design process of the VR game. First, a participatory design model was adopted to ensure the validity of the simulation game scenarios by experts from the field and to enhance acceptance for the tool itself. In iterations, the scenarios were tested with an expert group, and the results were fed back into the design of the next scenario. The test sessions included a pre-test-questionnaire (summarizing input-variables), a briefing by the experts of the situation within the simulation game, video-based observations of the test sessions (the process), a post-test questionnaire, and a debriefing phase focusing on both the play experience and the learning outcomes. The test sessions took place in experimental settings at a university, because the technological equipment was not sufficient at the workplace of the experts. The test groups were recruited from the experts available at the moment of the test, which was due to the flexible work schema of the experts. A minimum of two researchers were present as objective observers, without intervening in the game play process itself. Because of the limited number of experts in the field, the number of test persons available was also limited, which lead to a qualitative analysis of the data gathered from the tests.

Using questionnaires, the players were asked about positive and negative aspects of the game, regarding realism of the scenario, feelings of preparedness for future assignments, cooperation with team colleagues, and ability of recognizing deviant objects and behavior. In summary ${ }^{1}$, the players mentioned that interaction with other team colleagues, which was supported with the set-up, was one of the most important elements of the game that provided realistic feeling. On the other hand, the visuals of the environment, especially the limited animations and variations of the avatars, did not foster realism for the game, as reported by the players. Communication was mentioned as the most important feature of the game that supported the objective of the preparation of the player for future assignments. The limited number of triggers and details of the scenario did not support this goal in the experience of the players. For the cooperation with other colleagues, the communication possibilities of the game were positively noted. On the other hand, the players stated that they tended to be very much focused on the game play, which interfered with communication with other players. For the task of recognizing deviant objects and behaviors, players reported that the options of the game to hide objects, to show relationships between objects, and to drop objects in very unusual places was seen as an advantage for the simulation game. Disadvantages were seen in the limited sounds within the virtual environment and the limited animations of the avatars. Thus, functional fidelity was high and appreciated by the players, but physical fidelity was too low to enhance the experience of the players. Psychological fidelity, referred to as being focused on the assignment in the game, was also high, but

${ }^{1}$ For a more detailed design and analysis, see: Lukosch, van Ruijven, \& Verbraeck, 2012a, 2012b; Lukosch, van Nuland, van Ruijven, van Veen, \& Verbraeck, 2014. 
interfered with the communication aspects of the game. The outcomes of the first test lead us to develop of a second game scenario, aimed at a self-directed training activity without interaction with a trainer during game play.

In this case, functional fidelity was represented through the interaction and communication possibilities of the game scenarios and the role the trainer played and/or his absence from the game. Physical fidelity was represented by a detailed VR game environment. Psychological fidelity was represented by time pressure and communication, and the location of the players. It showed that the communication aspect had the highest impact on the acquisition of situational awareness within the teams. The training with the realistic game scenario that was based on an existing location was appreciated, especially for the training of novel team members, but did not include enough details to provide a realistic training situation. It seems that when an existing location is used as a reference for the simulation game environment, then every single detail has to show high physical fidelity. In summary, the qualitative evaluation showed that psychological and functional fidelity was more important for a positive player experience than physical fidelity, yet a lack of physical fidelity limited the experience of the players in the VR environment. As players mentioned that communication and exchange with other players was important for the learning experience, we conclude that in this case, a notion such as social fidelity plays an important role as well.

\subsection{AR Box Game: Forensic Teamwork}

In a project with the Dutch Police, we investigated how AR affects communication, information sharing, and situational awareness of geographically distributed teams in the safety and security domain (Lukosch et al., 2015). AR was used to remotely support local professionals at a crime scene by connecting them via AR with remote experts. AR allowed remote experts to see the crime scene and jointly annotate it with local professionals. We conducted a series of experiments using realistic scenarios at staged crime scenes with professionals from the safety and security domain to study the effect of AR. All experiment setups were of high fidelity. The physical fidelity was supported by the staged crime scene. The functional fidelity was high, as apart from the AR technology, only real equipment was used. High psychological and social fidelity was achieved by conducting the experiments with professionals only.

Such a high-fidelity setup is difficult to achieve with regard to staging the crime scene and involving professionals. Also, from earlier experiments at a realistic training site, we learned that a realistic situation is sometimes too complex to study technologies in rigorous ways (see for more details see e.g., Datcu et al. $(2014,2015)$ ). To study the effect of remote support via AR on coordination and communication of distributed teams in a more rigorous way, we thus designed the "AR Box game" to simplify the setup and complexity.

The "AR Box Game" is collaborative and mimics characteristics from a complex real scenario, but can also be played by non-professionals. For the game, we chose a scenario related to crime scene investigation. The game needs to be played by three players, one in the role of a remote police agent, and two as police agents investigating the crime scene.

At the crime scene, there are several boxes which can contain pieces of evidence (cf. Fig. 1, left). During the game, these pieces of evidence resemble blue, red, green, yellow and white Lego blocks. One of the local players has the skill to collect the 
evidence in the form of blue and red Lego blocks. The other local player can collect green and yellow Lego blocks. None of the players can collect a white Lego block; they are considered dangerous and possibly explosive.
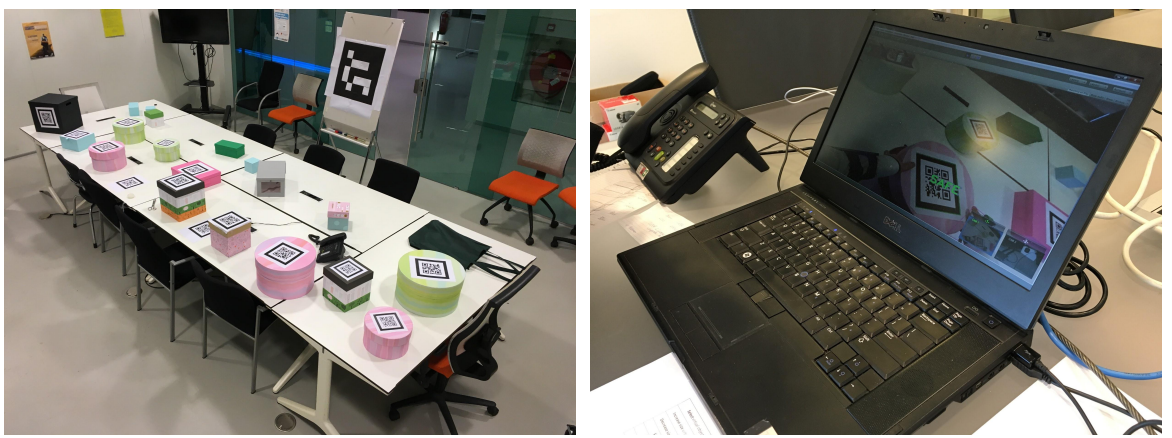

Fig. 1. The game setup for the local players (left) and the remote player (right)

The players either arrive at the crime scene at the same time and jointly investigate the crime scene, or they can do so sequentially. At the crime scene, it is their task to collect all non-dangerous pieces of evidence from the boxes. The local players are supported by a remote player who knows which boxes contain dangerous material and which do not. For this purpose, the local players share their view with remote player (cf. Fig. 1 right)

The goal of the game is to collect all evidence without opening one box containing a dangerous piece of evidence. Once all pieces of evidence are collected, the local players receive instructions from the remote player on how to combine the individual pieces of evidence, i.e., how to build a tower of Lego blocks showing a specific color pattern. If, during game play, a box containing a dangerous piece of evidence is opened, the game immediately ends and the players have not accomplished their task.

While playing, the players can exchange information in AR (for a detailed description of the underlying technology, refer to Datcu et al. (2016)). Only the remote player can see whether a box is dangerous or safe (cf. Fig. 2, left). Local and remote players can annotate boxes in AR e.g., to indicate the color of the block in the box or mark which boxes have already been emptied. The information the players leave behind on the boxes allows them to share information and coordinate their work (cf. Fig. 2, right). Local player(s) and the remote player can furthermore talk to each other during the whole game play using a continuously open audio channel.

Compared to earlier high-fidelity experiments, the "AR box game" is of lower fidelity. The physical fidelity only mimics a crime scene. The functional fidelity is also lower, as the necessary actions are also only mimicking the ones from a staged crime scene. The story of the game is also of lower psychological and social fidelity, as the game story is only linked to a crime scene and the lower physical fidelity of the crime scene has an impact on the psychological and social fidelity. 


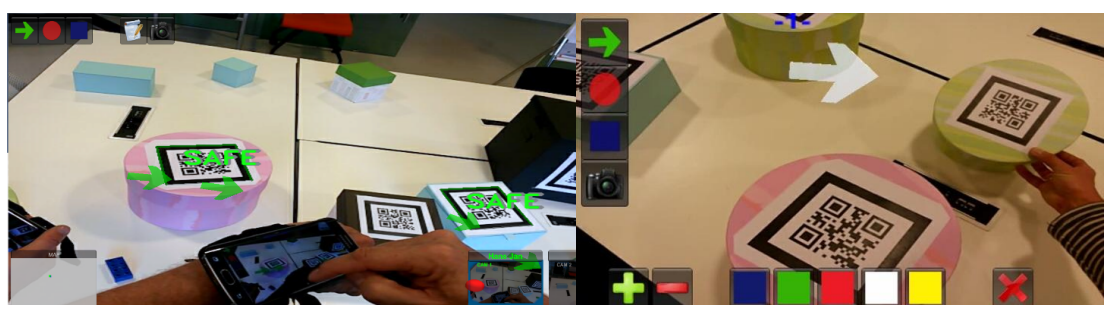

Fig. 2. View of the remote (left) and local player (right) showing information in AR

The game was tested with four professionals from the field to evaluate the realism and fidelity of the game. All professionals had participated in at least one of the high fidelity experiments above. The game was played several times, during which the professionals took on the different roles of local player or remote player. Also, rounds of synchronous investigation with two players at the same time at the local site as well as asynchronous investigation were played. The observation and debriefing showed that the game was able to mimic the situation of distributed work and remote support as in the high-fidelity experiments. The story explosives being hidden in some of the boxes was strong enough to make the players feel as if they were in a real, serious situation. One participant remarked after they failed one round and opened the wrong box that he was shocked as if, in reality, his colleague would have been injured based on his wrong information. This also shows that games combining the digital and physical world have the potential to provoke strong, compelling experiences. In summary, this shows that lower fidelity, here specifically physical and functional fidelity, can result in similar experiences as high-fidelity setups. Surprisingly, the simple story of dangerous content in one box resulted in a high psychological fidelity for one participant. Here, future research needs to investigate whether this is also true for non-professional players that might feel less responsible for local-player actions.

\subsection{Applied Games for Healthcare}

It is becoming increasingly commonplace to use games which utilize virtual reality technology for health-related topics. A common application of games in health care is to train health professionals. For example the game Night Shift, developed by Jesse Schell's team, was used to train physicians' decision making skills in emergency departments (Mohan et al., 2017). Healthcare training applications can be classified under the general umbrella of training applications and hence follow a similar development process as other applied games for the training of professionals, as described in the previous examples given above. In this section, we focus on the application of applied games for rehabilitation and therapeutic purposes.

Mirror therapy is an example where physical, functional and psychological fidelities interplay. Mirror therapy is used to help patients with phantom pain manage their symptoms, as well as to help patients with motor impairments after a stroke (Regenbrecht et al., 2012; Thieme et al., 2018). In its most basic form, mirror therapy uses an optical mirror to show one side of the patient as if it is the other side. The aim is to mirror the unimpaired side so that it creates the visual illusion that the missing or impaired side is fully functional again. 
Using an optical mirror to display a real hand of the patient can be considered the highest level of physical visual fidelity. However, when considering psychological fidelity, which also includes the experience of ownership of the virtual or mirrored limb, virtual reality technology might be able to elicit a stronger response (Hoermann, Franz, \& Regenbrecht, 2012; Regenbrecht, Hoermann, Ott, Müller, \& Franz, 2014).

Applied games which mimic some form of mirror therapy have been studied for their impact on motor rehabilitation (Laver, George, Thomas, Deutsch, \& Crotty, 2015). Regenbrecht et al. created Augmented Reflection Technology (ART), which was used for clinical and non-clinical studies (Hoermann et al., 2017; Regenbrecht, Franz, McGregor, Dixon, \& Hoermann, 2011; Regenbrecht, McGregor, et al., 2011). An augmented reality memory game, TheraMem, played by amplifying the movement of the impaired hand was used with patients in their chronic phase after stroke (Hoermann, Hale, Winser, \& Regenbrecht, 2014). TheraMem's physical fidelity of the virtual components can be described as low, with most of the virtual objects having only vague resemblance to real world objects. However, the psychological fidelity related to the feeling of being in control of their augmented real hands could be, based on the reports of many users, described as high.

The use of game technology for phantom pain management has also been studied (Rothgangel \& Bekrater-Bodmann, 2019). In their review paper, Rothgangel \& Bekrater-Bodmann pointed out that a number of studies have successfully created virtual and augmented reality applications. In particular, they pointed out that AR/VR technology can create virtual therapeutic environments beyond physical reality. For example for patients who have lost both their limbs, the representation of entirely virtual limbs without any real world counterpart is a necessity. Yet, even these virtual representations with an arguably low physical fidelity, aim to maximize the psychological fidelity i.e., the feeling of ownership and agency for the displayed limb. Several aspects that link virtual components of rehabilitation applications with the experience of presence were discussed by Schüler et al. (Schüler, Santos, \& Hoermann, 2015; Schüler, Ferreira dos Santos, \& Hoermann, 2014). In particular, they linked movement visualization, performance feedback and context information with presence dimensions. Movement visualization is linked to the way users are represented in virtual and augmented environments. The level of physical fidelity can, however, vary from fictive and abstract to anthropomorphic avatar representation, in either the first or the third-person perspective (Schüler et al., 2014).

Nevertheless, and necessary for all systems, is the need to provide feedback to patients about their performance and the results of their actions. In motor rehabilitation applications, patients need to be informed about the movements of their actual limbs as well as the results of these movements in the virtual environment e.g., did they grab the virtual diamond or catch the virtual butterfly (Jordan \& King, 2011; Lange et al., 2011). In augmented reality rehabilitation systems, this could be as simple as providing a video overlay of the actual hand over the virtual content and providing feedback about how well the hand posture matches the target posture (Pinches \& Hoermann, 2016). Feedback for patients on their movement performance and hence high functional fidelity of movement representation is essential for motor rehabilitation applications.

The third element, context information, is linked to realness perception and therefore, physical fidelity. This could be a very detailed virtual environment in which the patient performs therapeutic exercises, but can also be reduced to a minimum. In rehabilitation systems, this information can be used as a motivational aspect, but might not necessarily have a direct therapeutic impact. Hence, it is not just the physical fidelity 
of the context information which may be important, but how well this information matches the requirement of the task or exercise that the patient performs.

\section{Towards a Framework of Applied Game Fidelity}

In this section we summarize the results from the literature review, as well as our observations from the three cases illustrated above. As shown, the degree of realism of an applied game can be described in terms of a game's fidelity (Alexander, et al., 2005; Salas, Wilson, Burke, \& Priest, 2005; Harteveld, 2011), and as such has impact on the processes within, and the outcomes of, a game. The (shared) experience of playing an applied game enables actors to perceive and comprehend a problem or system of which they are a part, such as a police agent or forensic researcher's work environment, or a patient within a rehabilitation process. Applied games represent media that enable a new, or at least different, view of familiar situations. Individuals are often deeply embedded in a situation in which they are not able to take on the role of a critical, so called first order, observer; they develop a blind spot for the situation and environment in which they participate. Applied games represent media in the sense that they act as second-order observers (as they mediate what we observe) and help in overcoming the blind spot of first-order observers of the system and the individuals themselves (Luhmann, 1996). As such, applied games should be designed with the 'right' or at least a sufficient level of fidelity to serve as interactive second-order observer.

Related research shows that a higher level of fidelity does not necessarily lead to improved effects of an applied game. Yet, it seems to be hard to define the 'right' or at least sufficient level of fidelity for the design of applied games with a certain effect in mind. To help designers to better understand and assess the elements that constitute game fidelity, and that influence the game experience, we propose a framework of applied game fidelity that will have to be validated by case studies in the future. Table 1 summarizes the findings from literature and our cases so far.

\begin{tabular}{|c|c|c|c|}
\hline$\stackrel{\text { Low } \quad \text { High }}{\longrightarrow}$ & Game Characteristic & Affected Concepts & Game Experience \\
\hline $\begin{array}{l}\text { Psychological } \\
\text { Fidelity }\end{array}$ & $\begin{array}{l}\text { High accordance } \\
\text { with reality }\end{array}$ & $\begin{array}{l}\text { Emotion, } \\
\text { Experience }\end{array}$ & $\begin{array}{l}\text { Ownership, } \\
\text { Agency }\end{array}$ \\
\hline $\begin{array}{l}\text { Functional } \\
\text { Fidelity }\end{array}$ & $\begin{array}{l}\text { High accordance } \\
\text { with reality }\end{array}$ & $\begin{array}{l}\text { Tasks, } \\
\text { Procedures }\end{array}$ & Feedback \\
\hline $\begin{array}{l}\text { Physical } \\
\text { Fidelity }\end{array}$ & $\begin{array}{l}\text { Lower accordance } \\
\text { with reality }\end{array}$ & $\begin{array}{l}\text { Emulation, } \\
\text { Audio-visual } \\
\text { design }\end{array}$ & Recognition \\
\hline $\begin{array}{l}\text { Social } \\
\text { Fidelity }\end{array}$ & To be studied & $\begin{array}{l}\text { Interaction with } \\
\text { other players and } \\
\text { non-player } \\
\text { characters }\end{array}$ & Collaboration \\
\hline
\end{tabular}

Table 1: Proposed Framework of Interaction Fidelity for Applied Games 
Table 1 shows the four dimensions of fidelity. Each dimension can be defined on a continuum from low to high fidelity. The table illustrates that psychological fidelity is directly linked to high accordance with reality, is triggering emotions and experiences, leading to experienced ownership and agency. Functional fidelity also affects the outcomes of a game when high accordance with reality is reached. It is related to the representation of tasks and procedures in a game, leading to effective feedback. Contrary to often seen research hypotheses, physical fidelity can be expressed on a lower level of resemblance with reality. It represents an audio-visual emulation of the reference system, and is related to recognition of the physical game space. Social fidelity has still to be studied, and relates to the interaction with other players and nonplayable characters. It can lead to experienced collaboration. Further research will focus on the validation of the framework.

\section{Discussion and Conclusion}

As Bhargava et al., 2018 show, fidelity of a virtual environment did not have a significant impact on learning outcomes. The results show, contrary to common belief, that there is no linear relationship between level of realism and effectiveness of training. Abstraction and simplification can lead to excellent training outcomes (Toups, Kerne, and Hamilton, 2011).

Our own studies show that applied games that are designed to address the communication and interaction between team members contribute to the developmental process of situation awareness and communication. Thus, they are able to act as a second-order observer of systems and problems. A realistic VR of an applied game provides a shared experience to the players, and supports the perception of a situation. The environment plus information of other players enhances the individual comprehension of a situation. This study showed that communication and interaction of the players were two of the most important aspects, which we refer to as social fidelity. This fourth aspect, which has not yet been defined as such, will enrich the design of applied games with crucial social competencies individuals - as team members or not-nowadays need to actively participate in the systems of our networked society.

In summary, applied games require a high level of functional and psychological fidelity to be effective. While the appropriate and accurate provision of feedback to actions in the virtual environment (functional fidelity) is paramount, physical fidelity of the environment is less important. It can be speculated that a reduction of the physical fidelity of the environment to the bare minimum might help players focus on key aspects of the interventions, which in motor-rehabilitation games is to keep their attention on the observation or execution of movements, and in professional games to carry out realistic tasks, based on procedures of the reference system.

From our results, we can conclude that when learning is the goal of an applied game, physical fidelity does not have to be high in order to develop an effective tool. High fidelity games are usually costlier and more time consuming, which makes it a challenge to balance how much realism is needed in order to reach the intended effect. The target group and purpose of the game should carefully be evaluated against the cost of high fidelity, in order to design an effective game with a sufficient level of fidelity.

Further research should explore whether ethical or value fidelity could also be added to the framework, before the framework itself can be validated. When designing applied 
games, a game designer is influenced by his/her cultural background, experiences, and the expected outcomes of the game. Related values of the designer influence the design and application of a game, and the evaluation of its outcomes. Game designers should thus be aware of this influence, open to diversity and differing values of game players, and reflect on them. A diverse design team might limit the bias in this respect. An important aspect related to ethical fidelity is the responsibility of the game designer of an applied immersive game. In training games with sensitive content, or with vulnerable people as the target group, such as young, ill or elderly people, possible effects of the game should be considered in all stages of the design process. In an example of a game for humanitarian aid workers that was part of one of our research projects, some participants were affected by the high level of realism of the processes and reminded of impactful situations of field work. The game facilitators could not address this issue as they were not prepared for such reactions to the game. One approach to be explored in future research might be the notion of "value sensitive design" (van de Poel, 2013), where such possible effects and risks are evaluated during the whole process of designing an artifact, such as an applied immersive game.

\section{References}

1. Aarseth, E. J. (1997). Cybertext: perspectives on ergodic literature. Baltimore, MD, USA: Johns Hopkins University Press.

2. Alessi, S. M. (1988). Fidelity in the design of instructional simulations. Journal of Computer Based Instruction, 15(2), 40-47.

3. Alexander, A.L., Brunye, T., Sidman, J., Weil, S.A. (2005). From Gaming to Training: A Review of Studies on Fidelity, Immersion, Presence, and Buy-in and Their Effects on Transfer in PC-Based Simulations and Games. Aptima Inc, Woburn.

4. Bhargava, A., Bertrand, J. W., Gramopadhye, A. K., Madathil, K. C., \& Babu, S. V. (2018). Evaluating Multiple Levels of an Interaction Fidelity Continuum on Performance and Learning in Near-Field Training Simulations. IEEE Transactions on Visualization and Computer Graphics, 24(4), 1418-1427.

5. Brown, E., \& Cairns, P. (2004). A Grounded Investigation of Game Immersion. In CHI '04 Extended Abstracts on Human Factors in Computing Systems (pp. 1297-1300). New York, NY, USA: ACM.

6. Chalmers, A. \& Debattista, K. (2009). Level of Realism for Serious Games, IEEE Proceedings of 2009 Conference in Games and Virtual Worlds for Serious Applications.

7. Chalmers, A., Debattista, K., \& Ramic-Brkic, B. (2009). Towards high-fidelity multisensory virtual environments. The Visual Computer, 25(12), 1101.

8. Cornacchione Jr, E. B. (2012). Fidelity and game-based technology in management education. BAR-Brazilian Administration Review, 9(2), 147-167.

9. Datcu, D., Cidota, M., Lukosch, H., \& Lukosch, S. (2014). On the usability of augmented reality for information exchange in teams from the security domain. In Intelligence and Security Informatics Conference (JISIC) 2014 IEEE Joint, 160-167. IEEE.

10. Datcu, D., Lukosch, S., Lukosch, H., \& Cidota, M. (2015). Using augmented reality for supporting information exchange in teams from the security domain. Security Informatics, 4(1), 10.

11. Datcu, D.; Lukosch, S. G. \& Lukosch, H. K. (2016). Handheld Augmented Reality for Distributed Collaborative Crime Scene Investigation Proceedings of the 19th International Conference on Supporting Group Work, ACM, 267-276. 
12. Dwyer, F. M. (1974). Strategies for improving visual learning. University Park: Pennsylvania State University, Learning Services.

13. Feinstein, A. H., \& Cannon, H. M. (2002). Constructs of simulation evaluation. Simulation \& Gaming, 33(4), 425-440.

14. Gagne, R. M. (1954). Training devices and simulators: Some research issues. American Psychologist, 9, 95-107.

15. Galloway, A.R. (2004). Social Realism in Gaming. The International Journal of Computer Game Research, 4(1).

16. Harteveld, C. (2011). Triadic Game Design. Springer, London.

17. Harrison, S., Tatar, D., \& Sengers, P. (2007). The three paradigms of HCI. SIGCHI Conference on Human Factors in Computing Systems. April, San Jose, California, USA, 1-18.

18. Hays, R. T., \& Singer, M. J. (1989). Simulation fidelity as an organizing concept. In Simulation Fidelity in Training System Design, 47-75. Springer, New York, NY.

19. Hoermann, S., Ferreira dos Santos, L., Morkisch, N., Jettkowski, K., Sillis, M., Devan, H., Cutfield, N. J. (2017). Computerised mirror therapy with Augmented Reflection Technology for early stroke rehabilitation: clinical feasibility and integration as an adjunct therapy. Disability and Rehabilitation, 39(15), 1503-1514.

20. Hoermann, S., Franz, E. A., \& Regenbrecht, H. (2012). Referred Sensations Elicited by Video-Mediated Mirroring of Hands. PLOS ONE, 7(12), e50942.

21. Hoermann, S., Hale, L., Winser, S. J., \& Regenbrecht, H. (2014). Patient engagement and clinical feasibility of Augmented Reflection Technology for stroke rehabilitation. International Journal on Disability and Human Development, 13(3), 355-360.

22. Jordan, K., \& King, M. (2011). Augmented Reality Assisted Upper Limb Rehabilitation Following Stroke. Augmented Reality - Some Emerging Application Areas.

23. Kayali, F.; Luckner, N.; Purgathofer, P.; Spiel, K. \& Fitzpatrick, G. (2018). Design Considerations Towards Long-term Engagement in Games for Health Proceedings of the 13th International Conference on the Foundations of Digital Games, ACM, 35:1-35:8.

24. Lange, B., Chang, C., Suma, E., Newman, B., Rizzo, A. S., \& Bolas, M. (2011). Development and evaluation of low cost game-based balance rehabilitation tool using the microsoft kinect sensor. In 2011 Annual International Conference of the IEEE Engineering in Medicine and Biology Society (pp. 1831-1834).

25. Laver, K. E., George, S., Thomas, S., Deutsch, J. E., \& Crotty, M. (2015). Virtual reality for stroke rehabilitation. In Cochrane Database of Systematic Reviews. John Wiley \& Sons, Ltd.

26. Lombard, M., \& Ditton, T. (1997). At the heart of it all: The concept of presence. Journal of Computer-Mediated Communication, 3(2).

27. Lombard M., Ditton T.B., Crane D., \& Davis B. (2000). Measuring presence: A literature-based approach to the development of a standardized paper-and-pencil instrument. Proceedings of Presence 2000.

28. Luhmann, N. (1996). Die Realität der Massenmedien. Opladen: Westdeutscher Verlag.

29. Lukosch, H., van Ruijven, T., \& Verbraeck, A. (2012a). The participatory design of a simulation training game. In Proceedings of the winter simulation conference, 142.

30. Lukosch, H., van Ruijven, T., \& Verbraeck, A. (2012b). The other city-Designing a serious game for crisis training in close protection. In Conference Proceedings-9th International Conference on Information Systems for Crisis Response and Management.

31. Lukosch, H., van Nuland, B., van Ruijven, T., van Veen, L., \& Verbraeck, A. (2014). Building a virtual world for team work improvement. In Frontiers in Gaming Simulation, 60-68. Springer, Cham. 
32. Lukosch, S.; Lukosch, H.; Datcu, D. \& Cidota, M. (2015). Providing Information on the Spot: Using Augmented Reality for Situational Awareness in the Security Domain. Computer Supported Cooperative Work (CSCW) - The Journal of Collaborative Computing and Work Practices, 24, 613-664.

33. McMahan, R. (2011). Exploring the Effects of Higher-Fidelity Display and Interaction for Virtual Reality Games. Doctoral dissertation, Virginia Polytechnic Institute and State University.

34. McMahan, R. P., Bowman, D. A., Zielinski, D. J., \& Brady, R. B. (2012). Evaluating display fidelity and interaction fidelity in a virtual reality game. IEEE Transactions on Visualization \& Computer Graphics, (4), 626-633.

35. Martin, E. L., \&Waag,W. L. (1978). Contributions of platform motion to simulator training effectiveness: Study I-Basic contact. Brooks Air Force Base, TX: Air Force Human Resources Laboratory.

36. Miller, G. E. (1974). Some considerations in the design and utilization of simulators for technical training (Rep. No. AFHRL-TR-74-65). Alexandria, VA: Air Force Human Resource Laboratory.

37. Mohan, D., Farris, C., Fischhoff, B., Rosengart, M. R., Angus, D. C., Yealy, D. M., ... Barnato, A. E. (2017). Efficacy of educational video game versus traditional educational apps at improving physician decision making in trauma triage: randomized controlled trial. $B M J, 359, \mathrm{j} 5416$.

38. Pinches, J., \& Hoermann, S. (2016). Evaluating automated real time feedback and instructions during computerized mirror therapy for upper limb rehabilitation using augmented reflection technology. In P. M. Sharkey \& A. A. Rizzo (Eds.), Proceedings 11th International Conference on Disability, Virtual Reality \& Associated Technologies (pp. 65-72). Los Angeles, California, USA.

39. Regenbrecht, H., Franz, E. A., McGregor, G., Dixon, B. G., \& Hoermann, S. (2011). Beyond the Looking Glass: Fooling the Brain with the Augmented Mirror Box. Presence: Teleoperators and Virtual Environments, 20(6), 559-576.

40. Regenbrecht, H., Hoermann, S., McGregor, G., Dixon, B., Franz, E., Ott, C., Hoermann, J. (2012). Visual manipulations for motor rehabilitation. Computers \& Graphics, 36(7), 819-834.

41. Regenbrecht, H., Hoermann, S., Ott, C., Müller, L., \& Franz, E. (2014). Manipulating the Experience of Reality for Rehabilitation Applications. Proceedings of the IEEE, 102(2), 170-184.

42. Regenbrecht, H., McGregor, G., Ott, C., Hoermann, S., Schubert, T., Hale, L., ... Franz, E. (2011). Out of reach? - A novel AR interface approach for motor rehabilitation. In Mixed and Augmented Reality (ISMAR), 2011 10th IEEE International Symposium on (pp. 219-228). Basel, Switzerland: IEEE.

43. Rothgangel, A., \& Bekrater-Bodmann, R. (2019). Mirror therapy versus augmented/virtual reality applications: towards a tailored mechanism-based treatment for phantom limb pain. Pain Management.

44. Salas, E., Wilson, K.A., Burke, C.S., \& Priest, H. (2005). Using Simulation-Based Training to Improve Patient Safety: What Does It Take? Journal on Quality and Patient Safety, 31(7), 363-371.

45. Schraffenberger, H. \& Van der Heide, E. (2013). From coexistence to interaction: influences between the virtual and the real in augmented reality. Proceedings of the 19th International Symposium on Electronic Art, ISEA2013.

46. Schüler, T., Santos, L. F. dos, \& Hoermann, S. (2015). Designing virtual environments for motor rehabilitation: Towards a framework for the integration of best-practice information. In 2015 International Conference on Virtual Rehabilitation (ICVR) (pp. 145-146)

47. Schüler, Thomas, Ferreira dos Santos, L., \& Hoermann, S. (2014). Harnessing the experience of presence for virtual motor rehabilitation: Towards a guideline for the 
development of virtual reality environments. In Proceedings 10th International Conference on Disability, Virtual Reality \& Associated Technologies (pp. 373-376). Gothenburg, Sweden: The University of Reading, Reading, UK.

48. Thieme, H., Morkisch, N., Mehrholz, J., Pohl, M., Behrens, J., Borgetto, B., \& Dohle, C. (2018). Mirror therapy for improving motor function after stroke. Cochrane Database of Systematic Reviews, (7).

49. Toups, Z.O., Kerne, A. \& W.A. Hamilton (2011). The team coordination game: Zerofidelity simulation abstracted from fire emergency response practice. $A C M$ Transactions on Computer-Human Interaction 18(4), 37 pages.

50. Tun, J. K., Alinier, G., Tang, J., \& Kneebone, R. L. (2015). Redefining simulation fidelity for healthcare education. Simulation \& Gaming, 46(2), 159-174.

51. Van de Poel, I. (2013). Translating values into design requirements. In Philosophy and engineering: Reflections on practice, principles and process, 253-266. Springer, Dordrecht.

52. Waller, D., Hunt, E., \& Knapp, D. (1998). The Transfer of Spatial Knowledge in Virtual Environment Training. Presence: Teleoperators and Virtual Environments, 7(2):129-143, 1998. 